\title{
SPECTRAL AREA ESTIMATES FOR NORMS OF COMMUTATORS
}

\author{
Muneo $\mathrm{Cho}^{*}{ }^{*}$ and TakahiKo NaKazi ${ }^{* *}$
}

\begin{abstract}
Let $A$ and $B$ be commuting bounded linear operators on a Hilbert space. In this paper, we study spectral area estimates for norms of $A^{*} B-B A^{*}$ when $A$ is subnormal or $p$-hyponormal.
\end{abstract}

\section{Introduction}

Let $\mathcal{H}$ be a Hilbert space and $\mathcal{B}(\mathcal{H})$ the set of all bounded linear operators on $\mathcal{H}$. If $T$ is a hyponormal operator in $\mathcal{B}(\mathcal{H})$ then C. R. Putnam [7] proved that $\left\|T^{*} T-T T^{*}\right\| \leq \operatorname{Area}(\sigma(T)) / \pi$, where $\sigma(T)$ is the spectrum of $T$. The second named author [5] has proved that if $T$ is a hyponormal operator and $K$ is in $\mathcal{B}(\mathcal{H})$ with $K T=T K$ then

$$
\left\|T^{*} K-K T^{*}\right\| \leq 2\{\operatorname{Area}(\sigma(T)) / \pi\}^{1 / 2}\|K\| .
$$

We don't know whether the constant 2 in the inequality is best possible for a hyponormal operator. In $\S 2$, we show that the constant is not best possible for a subnormal operator.

When $T$ is a $p$-hyponormal operator in $\mathcal{B}(\mathcal{H})$, A. Uchiyama [10] generalized the Putnam inequality, that is,

$$
\left\|T^{*} T-T T^{*}\right\| \leq \phi\left(\frac{1}{p}\right)\|T\|^{2(1-p)}\{\operatorname{Area}(\sigma(T)) / \pi\}^{p} .
$$

This inequality gives the Putnam inequality when $p=1$. In $\S 3$, we generalize the above inequality for the spectral area estimate of $\left\|T^{*} K-K T^{*}\right\|$ when $T K=K T$. H. Alexander [1] proved the following inequality for a uniform algebra $A$. If $f$ is in $A$ then

$$
\operatorname{dist}(\bar{f}, A) \leq\{\operatorname{Area}(\sigma(f)) / \pi\}^{1 / 2} .
$$

Received January 25, 2006.

2000 Mathematics Subject Classification. Primary 47A20.

Key words and phrases. subnormal, p-hyponormal, Putnam inequality.

* This research is partially supported by Grant-in-Aid Scientific Research No.17540139.

** This research is partially supported by Grant-in-Aid Scientific Research No.17540176. 
The second named author [5] gave an operator version for the Alexander inequality. This was used in order to estimate $\left\|T^{*} K-K T^{*}\right\|$ when $T$ is a hyponormal operator and $K T=T K$. We also give an Alexander inequality for a $p$-hyponormal and we use it to estimate $\left\|T^{*} K-K T^{*}\right\|$.

In $\S 4$, we try to estimate $\left\|T^{*} K-K T^{*}\right\|$ for arbitrary contraction. In $\S 5$, we show a few results about area estimates for $p$-quasihyponormal operators, restricted shifts and analytic Toeplitz operators.

For $0<p \leq 1, T$ is said to be $p$-hyponormal if $\left(T^{*} T\right)^{p}-\left(T T^{*}\right)^{p} \geq 0$. A 1-hyponormal operator is hyponormal. For an algebra $\mathcal{A}$ in $\mathcal{B}(\mathcal{H})$, let lat $\mathcal{A}$ be the lattice of all $\mathcal{A}$-invariant projections. For a compact subset $X$ in $\ell, \operatorname{rat}(X)$ denotes the set of all rational functions on $X$.

\section{Subnormal operators}

In order to prove Theorem 1, we use the original Alexander inequality.

Theorem 1. Let $T$ be a subnormal operator in $\mathcal{B}(\mathcal{H})$ and $f$ a rational function on $\sigma(T)$ whose poles are not on it. Then

$$
\left\|T^{*} f(T)-f(T) T^{*}\right\| \leq\{\operatorname{Area}(\sigma(T)) / \pi\}^{1 / 2}\{\operatorname{Area}(\sigma(f(T))) / \pi\}^{1 / 2} .
$$

Proof. Suppose that $N \in \mathcal{B}(\mathcal{K})$ is a normal extension of $T \in \mathcal{B}(\mathcal{H})$ and $P$ is an orthogonal projection from $\mathcal{K}$ to $\mathcal{H}$. Then $T=P N \mid \mathcal{H}$ and so

$$
\begin{aligned}
& T^{*} f(T)-f(T) T^{*} \\
= & P N^{*} P f(N) P-P f(N) P N^{*} P \\
= & P N^{*} f(N) P-P f(N) P N^{*} P \\
= & P f(N) N^{*} P-P f(N) P N^{*} P \\
= & P f(N)(1-P) N^{*} P \\
= & P f(N)(1-P) \cdot(1-P) N^{*} P
\end{aligned}
$$

because $f(N) P=P f(N) P$ and $f(N) N^{*}=N^{*} f(N)$.

Let $F$ be a rational function in $\operatorname{rat}(\sigma(T))$. Put $\mathcal{B}_{F}=$ the norm closure of $\left\{g(F(N)) ; g \in \operatorname{rat}(\sigma(F(N))\}\right.$ then $P$ belongs to lat $\mathcal{B}_{F}$. Hence

$$
\begin{aligned}
& \left\|(1-P) F(N)^{*} P\right\| \\
\leq \quad & \operatorname{dist}\left(F(N)^{*}, \mathcal{B}_{F}\right) \leq \operatorname{dist}(\bar{z}, \operatorname{rat}(\sigma(F(N)))) \\
\leq \quad & \{\operatorname{Area}(\sigma(F(N))) / \pi\}^{1 / 2}
\end{aligned}
$$

by the Alexander's theorem [1]. Hence, applying $F$ to $F=z$ or $F=f$

$$
\begin{aligned}
& \left\|T^{*} f(T)-f(T) T^{*}\right\| \\
\leq & \left\|(1-P) f(N)^{*} P\right\| \cdot\left\|(1-P) N^{*} P\right\| \\
\leq & \{\operatorname{Area}(\sigma(f(N))) / \pi\}^{1 / 2}\{\operatorname{Area}(\sigma(N)) / \pi\}^{1 / 2} \\
\leq & \{\operatorname{Area}(\sigma(f(T))) / \pi\}^{1 / 2}\{\operatorname{Area}(\sigma(T)) / \pi\}^{1 / 2} .
\end{aligned}
$$


If $T$ is a cyclic subnormal operator and $K T=T K$ then using a theorem of T. Yoshino [12] we can prove that

$$
\left\|T^{*} K-K T^{*}\right\| \leq\{\operatorname{Area}(\sigma(T)) / \pi\}^{1 / 2}\{\operatorname{Area}(\sigma(K)) / \pi\}^{1 / 2} .
$$

The proof is almost same to one of Theorem 1 .

\section{3. p-hyponormal operators}

In order to prove Theorem 2, we use an operator version of the Alexander inequality for a $p$-hyponormal operator. Unfortunately Lemma 3 is not best possible for $p=1$ (see [5]). Lemma 1 is due to W. Arveson [2, Lemma 2] and Lemma 2 is due to A. Uchiyama [11, Theorem 3].

We need the following notation to give Theorem 2 and Proposition 1 . Let $\phi$ be a positive function on $(0, \infty)$ such that

$$
\phi(t)=\left\{\begin{array}{cl}
t & \text { if } t \text { is an integer } \\
t+2 & \text { if } t \text { is not an integer. }
\end{array}\right.
$$

We write $\ell^{2} \otimes \mathcal{H}$ for the Hilbert space direct sum $\mathcal{H} \oplus \mathcal{H} \oplus \cdots$, and $1 \otimes T$ denotes the operator $T \oplus T \oplus \cdots \in \mathcal{B}\left(\ell^{2} \otimes \mathcal{H}\right)$ for each operator $T \in \mathcal{B}(\mathcal{H})$.

Lemma 1. Let $\mathcal{A}$ be an arbitrary ultra-weakly closed subalgebra of $\mathcal{B}(\mathcal{H})$ containing 1 , and let $T \in \mathcal{B}(\mathcal{H})$. Then

$$
\operatorname{dist}(T, \mathcal{A})=\sup \{\|(1-P)(1 \otimes T) P\| ; P \in \operatorname{lat}(1 \otimes \mathcal{A})\} .
$$

Lemma 2. If $T$ is a p-hyponormal operator, then

$$
\left\|T^{*} T-T T^{*}\right\| \leq \phi\left(\frac{1}{p}\right)\|T\|^{2(1-p)}\{\operatorname{Area}(\sigma(T)) / \pi\}^{p} .
$$

Lemma 3. If $T$ is a p-hyponormal operator then

$$
\operatorname{dist}\left(T^{*}, \mathcal{A}\right) \leq \sqrt{2 \phi\left(\frac{1}{p}\right)}\|T\|^{1-p}\{\operatorname{Area}(\sigma(T)) / \pi\}^{p / 2},
$$

where $\mathcal{A}$ is the strong closure of $\{f(T) ; f \in \operatorname{rat}(\sigma(T))\}$.

Proof. Let $S=1 \otimes T$. Then $S$ is $p$-hyponormal. In order to prove the lemma, by Lemma 1 it is enough to estimate $\sup \{\|(1-P) S P\| ; P \in \operatorname{lat}(1 \otimes \mathcal{A})\}$. If $P \in \operatorname{lat}(1 \otimes \mathcal{A})$ then $S P=P S P$ and so

$$
\begin{aligned}
& \|(1-P) S P\|^{2} \\
= & \left\|P S S^{*} P-P S P S^{*} P\right\| \\
= & \left\|P S S^{*} P-P S^{*} S P+P S^{*} S P-P S P S^{*} P\right\| \\
\leq & \left\|P\left(S^{*} S-S S^{*}\right) P\right\|+\left\|(P S P)^{*}(P S P)-(P S P)(P S P)^{*}\right\| \\
\leq & \left\|S^{*} S-S S^{*}\right\|+\left\|(P S P)^{*}(P S P)-(P S P)(P S P)^{*}\right\| .
\end{aligned}
$$


By $[11$, Lemma 4], $P S P$ is $p$-hyponormal and so by Lemma 2 we have

$$
\begin{aligned}
& \left\|P S S^{*} P-P S P S^{*} P\right\|^{2} \\
\leq & \phi\left(\frac{1}{p}\right)\|T\|^{2(1-p)}\{\operatorname{Area}(\sigma(T)) / \pi\}^{p} \\
& +\phi\left(\frac{1}{p}\right)\|P S P\|^{2(1-p)}\{\operatorname{Area}(\sigma(P S P)) / \pi\}^{p} \\
\leq & 2 \phi\left(\frac{1}{p}\right)\|T\|^{2(1-p)}\{\operatorname{Area}(\sigma(T)) / \pi\}^{p}
\end{aligned}
$$

because $\|P S P\| \leq\|S\|=\|T\|$ and $\sigma(P S P) \subset \sigma(S)=\sigma(T)$. By Lemma 1,

$$
\operatorname{dist}\left(T^{*}, \mathcal{A}\right) \leq \sqrt{2 \phi\left(\frac{1}{p}\right)}\|T\|^{1-p}\{\operatorname{Area}(\sigma(T)) / \pi\}^{p / 2} .
$$

Theorem 2. If $T$ is a p-hyponormal operator in $\mathcal{B}(\mathcal{H})$ and if $K$ is in $\mathcal{B}(\mathcal{H})$ with $K T=T K$, then

$$
\left\|T^{*} K-K T^{*}\right\| \leq 2 \sqrt{2 \phi\left(\frac{1}{p}\right)}\|T\|^{1-p}\{\operatorname{Area}(\sigma(T)) / \pi\}^{p / 2}\|K\| .
$$

Proof. When $\mathcal{A}$ is the strong closure of $\{f(T) ; f \in \operatorname{rat}(\sigma(T))\}$, for any $A \in \mathcal{A}$

$$
\left\|T^{*} K-K T^{*}\right\|=\left\|\left(T^{*}-A\right) K+A K-K T^{*}\right\| \leq 2\left\|T^{*}-A\right\|\|K\| .
$$

Now Lemma 3 implies the theorem.

In Theorem 2, if $p=1$, that is, $T$ is hyponormal then $\left\|T^{*} K-K T^{*}\right\| \leq$ $2 \sqrt{2}\{\operatorname{Area}(\sigma(T)) / 2\}^{1 / 2}\|K\|$. The constant $2 \sqrt{2}$ is not best because the second author [5] proved that $\left\|T^{*} K-K T^{*}\right\| \leq 2\{\operatorname{Area}(\sigma(T)) / 2\}^{1 / 2}\|K\|$. If $p=\frac{1}{2}$, that is, $T$ is semi-hyponormal then

$$
\left\|T^{*} K-K T^{*}\right\| \leq 4\|T\|^{1 / 2}\{\operatorname{Area}(\sigma(T)) / \pi\}^{1 / 4}\|K\| .
$$

\section{Norm estimates}

In general, it is easy to see that $\left\|T^{*} T-T T^{*}\right\| \leq\|T\|^{2}$. By Theorem 1 , if $T$ is subnormal and $f$ is an analytic polynomial then

$$
\left\|T^{*} f(T)-f(T) T^{*}\right\| \leq\|T\|\|f(T)\| .
$$

In this section, we will prove that $\left\|T^{*} T^{n}-T^{n} T^{*}\right\| \leq\|T\|^{n+1}$ for arbitrary $T$ in $\mathcal{B}(\mathcal{H})$.

Theorem 3. If $T$ is a contraction on $\mathcal{H}$ and $f$ is an analytic function on the closed unit disc $\bar{D}$ then $\left\|T^{*} f(T)-f(T) T^{*}\right\| \leq \sup _{z \in D}|f(z)|$. 
Proof. By a theorem of Sz.-Nagy [6], there exists a unitary operator $U$ on $\mathcal{K}$ such that $\mathcal{K}$ is a Hilbert space with $\mathcal{K} \supseteq \mathcal{H}$ and $T^{n}=P U^{n} \mid \mathcal{K}$ for $n \geq 0$, where $P$ is an orthogonal projection from $\mathcal{K}$ to $\mathcal{H}$. Then it is known that $U^{*} P=P U^{*} P$ and $f(T)=P f(U) \mid \mathcal{H}$. Hence

$$
\begin{aligned}
& T^{*} f(T)-f(T) T^{*} \\
= & P U^{*} P f(U) P-P f(U) P U^{*} P \\
= & P U^{*} P f(U) P-P f(U) U^{*} P \\
= & P U^{*}(I-P) f(U) P
\end{aligned}
$$

because $U^{*} P=P U^{*} P$ and $f(U) U^{*}=U^{*} f(U)$. Therefore

$$
\begin{aligned}
& \left\|T^{*} f(T)-f(T) T^{*}\right\| \\
= & \left\|P U^{*}(I-P) f(U) P\right\| \leq \sup _{z \in D}|f(z)| .
\end{aligned}
$$

Corollary 1. If $T$ is in $\mathcal{B}(\mathcal{H})$ then for any $n \geq 1\left\|T^{*} T^{n}-T^{n} T^{*}\right\| \leq\|T\|^{n+1}$.

Proof. Put $A=T /\|T\|$ then $A$ is a contraction and so by Theorem 2 $\left\|A^{*} A^{n}-A^{n} A^{*}\right\| \leq 1$ and so $\left\|T^{*} T^{n}-T^{n} T^{*}\right\| \leq\|T\|^{n+1}$.

\section{Remarks}

In this section, we give spectral area estimates for $p$-quasihyponomal operators, restricted shifts and analytic Toeplitz operators.

For $0<p \leq 1, T$ is said to be $p$-quasihyponormal if $T^{*}\left\{\left(T^{*} T\right)^{p}-\left(T T^{*}\right)^{p}\right\} T \geq$ 0 . A 1-quasihyponormal operator is called quasihyponormal.

Lemma 4. Let $T$ be p-quasihyponormal and $P$ be a projection such that $T P=$ PTP. Then PTP is also p-quasihyponormal.

Proof. Since $T$ is $p$-quaihyponormal, $T^{*}\left(T^{*} T\right)^{p} T \geq T^{*}\left(T T^{*}\right)^{p} T$. Hence, we have

$$
P T^{*}\left(T^{*} T\right)^{p} T P \geq P T^{*}\left(T T^{*}\right)^{p} T P .
$$

Since by the Hansen's inequality [4]

$$
\begin{aligned}
& P T^{*}\left(T^{*} T\right)^{p} T P=(P T P)^{*} P\left(T^{*} T\right)^{p} P(P T P) \\
\leq & (P T P)^{*}\left(P T^{*} T P\right)^{p}(P T P) \\
= & (P T P)^{*}\left\{(P T P)^{*}(P T P)\right\}^{p}(P T P)
\end{aligned}
$$

and by $0<p<1$

$$
\begin{aligned}
& P T^{*}\left(T T^{*}\right)^{p} T P \geq\left(P T^{*} P\right)\left(T P T^{*}\right)^{p}(P T P) \\
= & (P T P)^{*}\left\{(P T P)(P T P)^{*}\right\}^{p}(P T P),
\end{aligned}
$$

we have

$$
(P T P)^{*}\left\{(P T P)^{*}(P T P)\right\}^{p} \geq(P T P)^{*}\left\{(P T P)(P T P)^{*}\right\}^{p}(P T P) .
$$

Hence, $P T P$ is $p$-quasihyponormal. 
Proposition 1. If $T$ is a p-quasihyponormal operator in $\mathcal{B}(\mathcal{H})$ and if $K$ is in $\mathcal{B}(\mathcal{H})$ with $K T=T K$, then

$$
\left\|T^{*} K-K T^{*}\right\| \leq 4\left[\phi\left(\frac{1}{p}\right)\right]^{1 / 4}\|T\|^{1-p / 2}\{\operatorname{Area}(\sigma(T)) / \pi\}^{p / 4}\|K\| .
$$

In particular, if $T$ is quasihyponormal then

$$
\left\|T^{*} K-K T^{*}\right\| \leq 4\|T\|^{1 / 2}\{\operatorname{Area}(\sigma(T)) / \pi\}^{1 / 4}\|K\| .
$$

Proof. We can prove it as in the proof of Theorem 2. By [11, Theorem 6], $\left\|T^{*} T-T T^{*}\right\| \leq 2\|T\|^{2-p} \sqrt{\phi\left(\frac{1}{p}\right)}\{\operatorname{Area}(\sigma(T)) / \pi\}^{p / 2}$. Hence by Lemma 4

$$
\operatorname{dist}\left(T^{*}, \mathcal{A}\right) \leq 2\|T\|^{1-\frac{p}{2}} \phi\left(\frac{1}{p}\right)^{\frac{1}{4}}\{\operatorname{Area}(\sigma(T)) / \pi\}^{p / 4} .
$$

This implies the proposition.

Let $H^{2}$ and $H^{\infty}$ be the usual Hardy spaces on the unit circle and $z$ the coordinate function. $M$ denotes an invariant subspace of $H^{2}$ under the multiplication by $z$. By the well known Beurling theorem, $M=q H^{2}$ for some inner function. Suppose $N$ is the orthogonal complement of $M$ in $H^{2}$. For a function $\phi$ in $H^{\infty}, S_{\phi}$ is an operator on $N$ such that $S_{\phi} f=P(\phi f)(f \in N)$, where $P$ is the orthogonal projection from $H^{2}$ to $N$. For a symbol $\phi$ in $L^{\infty}, T_{\phi}$ denotes the usual Toeplitz operator on $H^{2}$.

Proposition 2. Suppose $\Phi=q \bar{\phi}$ belongs to $H^{\infty}$. Then

(1) $\left\|S_{\phi}^{*} S_{\phi}-S_{\phi} S_{\phi}^{*}\right\| \leq \operatorname{Area}(\overline{\Phi(D)}) / \pi$;

(2) $\left\|S_{\phi}^{*} S_{\phi}^{n}-S_{\phi}^{n} S_{\phi}^{*}\right\| \leq\{\operatorname{Area}(\overline{\Phi(D)}) / \pi\}^{n+1}$ for $n \geq 0$.

Proof. By a well known theorem of Sarason [8],

$$
\left\|S_{\phi}\right\|=\left\|\phi+q H^{\infty}\right\|=\left\|\bar{q} \phi+H^{\infty}\right\|=\left\|\bar{\Phi}+H^{\infty}\right\| .
$$

By Nehari's theorem [6], $\left\|\bar{\Phi}+H^{\infty}\right\|=\left\|H_{\bar{\Phi}}\right\|$, where $H_{\bar{\Phi}}$ denotes a Hankel operator from $H^{2}$ to $\bar{z} \bar{H}^{2}$. Since $\left\|H_{\bar{\Phi}}\right\|^{2}=\left\|T_{\Phi}^{*} T_{\Phi}-T_{\Phi} T_{\Phi}^{*}\right\|$, where $T_{\Phi}$ denotes a Toeplitz operator on $H^{2}$, by the Putnam inequality

$$
\left\|T_{\Phi}^{*} T_{\Phi}-T_{\Phi} T_{\Phi}^{*}\right\| \leq \operatorname{Area}\left(\sigma\left(T_{\Phi}\right)\right) / \pi=\operatorname{Area}(\overline{\Phi(D)}) / \pi .
$$

Now since $\left\|S_{\phi}^{*} S_{\phi}-S_{\phi} S_{\phi}^{*}\right\| \leq\left\|S_{\phi}\right\|^{2}$, (1) follows. (2) is also clear by the proof above and Corollary 1.

Proposition 3. Suppose $f$ and $g$ are in $H^{\infty}$. Then

$$
\left\|T_{f}^{*} T_{g}-T_{g} T_{f}^{*}\right\| \leq\{\operatorname{Area}(\overline{f(D)}) / \pi\}^{1 / 2}\{\operatorname{Area}(\overline{g(D)}) / \pi\}^{1 / 2} .
$$


Proof. It is easy to see that $T_{f}^{*} T_{g}-T_{g} T_{f}^{*}=H_{\bar{g}}^{*} H_{\bar{f}}$. Hence

$$
\left\|T_{f}^{*} T_{g}-T_{g} T_{f}^{*}\right\| \leq\left\|H_{\bar{g}}\right\| \cdot\left\|H_{\bar{f}}\right\| .
$$

Since $H_{\tilde{f}}^{*} H_{\bar{f}}=T_{f}^{*} T_{f}-T_{f} T_{f}^{*}$, by the Putnam inequality

$$
\left\|T_{f}^{*} T_{g}-T_{g} T_{f}^{*}\right\| \leq\{\operatorname{Area}(\overline{f(D)}) / \pi\}^{1 / 2}\{\operatorname{Area}(\overline{g(D)}) / \pi\}^{1 / 2} .
$$

\section{References}

[1] H. Alexander, Projections of polynomial hulls, J. Funct. Anal. 13 (1973), 13-19.

[2] W. Arveson, Interpolation problems in nest algebras, J. Funct. Anal. 20 (1975), no. 3, 208-233.

[3] M. Chō and M. Itoh, Putnam's inequality for p-hyponormal operators, Proc. Amer. Math. Soc. 123 (1995), no. 8, 2435-2440.

[4] F. Hansen, An operator inequality, Math. Ann. 246 (1979/80), no. 3, 249-250.

[5] T. Nakazi, Complete spectral area estimates and self-commutators, Michigan Math. J. 35 (1988), no. 3, 435-441.

[6] Z. Nehari, On bounded bilinear forms, Ann. of Math. (2) 65 (1957), 153-162.

[7] C. R. Putnam, An inequality for the area of hyponormal spectra, Math. Z. 116 (1970), 323-330.

[8] D. Sarason, Generalized interpolation in $H^{\infty}$, Trans. Amer. Math. Soc. 127 (1967), 179-203.

[9] B. Sz.-Nagy and C. Foias, Harmonic Analysis Of Operators On Hilbert Space, American Elsevier, New York, 1970.

[10] A. Uchiyama, Berger-Shaw's theorem for p-hyponormal operators, Integral Equations Operator Theory 33 (1999), 221-230.

[11] _ Inequalities of Putnam and Berger-Shaw for p-quasihyponormal operators, Integral Equations Operator Theory 34 (1999), no. 2, 91-106.

[12] T. Yoshino, Subnormal operators with a cyclic vector, Tohoku Math. (2) 21 (1969), 47-55.

MUNEO CHō

DEPARTMENT OF MATHEMATICS

KANAGAWA UNIVERSITY

JAPAN

E-mail address: chiyom010kanagawa-u.ac.jp

TAKAHIKO NAKAZI

DEPARTMENT OF MATHEMATICS

FACULTY OF SCIENCE

HOKKAIDO UNIVERSITY

SAPPORO 060-0810, JAPAN

E-mail address: nakazi@math.sci.hokudai.ac.jp 